\title{
IDENTIFICATION OF DISCRETE CHOICE DYNAMIC PROGRAMMING MODELS WITH NONPARAMETRIC DISTRIBUTION OF UNOBSERVABLES
}

\author{
LE-Yu CHEN \\ Academia Sinica
}

\begin{abstract}
This paper presents semiparametric identification results for the Rust (1994) class of discrete choice dynamic programming (DCDP) models. We develop sufficient conditions for identification of the deep structural parameters for the case where the per-period utility function ascribed to one choice in the model is parametric but the distribution of unobserved state variables is nonparametric. The proposed identification strategy does not rely on availability of the terminal period data and can therefore be applied to infinite horizon structural dynamic models. Identifying power comes from assuming that the agent's per-period utilities admit continuous choice-specific state variables that are observed with sufficient variation and satisfy certain conditional independence assumptions on the joint time series of observables. These conditions allow us to formulate exclusion restrictions for identifying the primitive structural functions of the model.
\end{abstract}

\section{INTRODUCTION}

Estimation of discrete choice dynamic programming (DCDP) models has become increasingly important in empirical economic research and in practical policy assessment. Excellent surveys of applications and estimation techniques may be found in Eckstein and Wolpin (1989), Rust (1994), Miller(1997), Keane and Wolpin (2009), and Aguirregabiria and Mira (2010). In this literature, however, underlying structural objects such as per-period utilities, transition laws of state variables and the joint distribution of unobservables are usually parametrically specified, thus necessarily risking over-restriction and exacerbating misspecification. Consequently, attention has turned to nonparametric specification.

This paper is a significant revision of my earlier Cemmap working papers (Chen (2007, 2009)) and the first chapter of my doctoral thesis (Econometric Inference Involving Discrete Indicator Functions, 2009, University College London). I thank my advisor Sokbae Lee for his encouragement and insightful advice on this research work. I am grateful to the co-editor and two anonymous referees for valuable comments and suggestions on previous versions of the paper. I am indebted to the editor Peter Phillips for constructive advice and comments which have considerably improved the presentation of this paper. I also benefited from helpful comments provided by Richard Blundell, Andrew Chesher, Hidehiko Ichimura, Oliver Linton, Pedro Mira, Lars Nesheim, Adam Rosen, and seminar participants at Aarhus, University of St. Gallen, Alicante, Academia Sinica, UCL/Cemmap and 2009 Far East and South Asia Meeting of the Econometric Society. Address correspondence to Le-Yu Chen, Institute of Economics, Academia Sinica, Nankang, Taipei, 115, Taiwan; e-mail: lychen@econ.sinica.edu.tw. 
In turn, new issues have arisen such as the inherent difficulty of identifying DCDP models nonparametrically, as emphasized by the influential work of Rust (1994, pp. 3127-3130). Magnac and Thesmar (2002) extended Rust's framework and indicated how identification can be achieved under bespoke specification of the joint distribution of unobservables and the functional forms of agents' preferences. However, the economic theory behind DCDP models is generally silent about distribution of the unobservables. Therefore, serious concerns of robustness arise and prompt interest in flexible specification of the distribution of unobservables. The present paper addresses this issue and establishes identification results for DCDP models of the Rust (1994) type allowing for a nonparametrically specified distribution of the unobserved state variables.

As noted in Magnac and Thesmar (2002), the class of Rust's DCDP models remains unidentified even if the joint distribution of unobservables is known. To secure identification, the researcher needs to further assume a functional form for the per-period utility of one of the choices. Therefore, throughout this paper, our identification analysis is semiparametric in the sense that one of the choicewise per-period utility functions is known a priori. Using this assumption, we show that the DCDP model with nonparametric unobservables can be identified under the following two key conditions: (i) the agent's per-period utilities admit continuous choice-specific state variables $z_{t}$ that are observed with sufficient variation and (ii) the joint time series of $z_{t}$ and the remaining observed state variables $w_{t}$ satisfy an empirically testable conditional independence assumption. These conditions enable us to form exclusion restrictions that provide identifying power when information about distribution of the unobservables is not available. Therefore, in this respect, we establish semiparametric identification of the dynamic counterpart of the static discrete choice model in which preference shock distributions are nonparametric but systematic utility functions are restricted to a certain function space. ${ }^{1}$

There is little literature addressing the problem of identifying structural dynamic discrete choice models with nonparametric unobservables. Our work is related to the identification analysis of Heckman and Navarro (2007). Their paper considers semiparametric identification of finite horizon dynamic optimal stopping models. Their setup allows for richer time series dependence between nonparametric unobservables. However, they assume that future values of some observed continuous state variables appear in the agent's current information set so that they can use the identification-at-infinity strategy in a fashion similar to the one entertained by Taber (2000). By contrast, we consider identification of the DCDP models in which all choices can be recurrent and the agent's information set contains only current and past realized state variables. We follow Rust's (1994) conditional independence framework to model the evolution of the state variables.

We develop an identification strategy that is applicable to both finite and infinite horizon DCDP models. Note that there is no terminal period for the infinite horizon model. Admitting the terminal period induces nonstationarity in the model so that one can gain extra identifying power by discriminating the 
model structure that varies across periods. For instance, Taber (2000), Heckman and Navarro (2007), and Aguirregabiria (2010) exploited the static nature of the agent's terminal period choice problem so that they can identify the terminal period structural parameters first and then solve the previous period problems via backward induction. Lacking the terminal period data poses a challenge in identification. This paper studies the use of exclusion restrictions to circumvent such identification problems and develops positive results for semiparametrically identifying all the deep structural parameters of Rust's DCDP model with nonparametric unobserved state variables. ${ }^{2}$

We now discuss other related literature in addition to the papers cited above. For the DCDP model with known distribution of unobserved state variables, Hotz and Miller (1993) established that structural value differences can be nonparametrically identified using the conditional choice probabilities and distribution of the unobservables. This insight has been exploited in Magnac and Thesmar's (2002) identification analysis and in the literature of the two-step estimation approach to reduce the computational complexity that arises from solving the fixed-point problem of the model. For two-step estimators of the DCDP model, see, e.g., Hotz and Miller (1993), Hotz, Miller, Sanders, and Smith (1994), Aguirregabiria and Mira (2002), and Srisuma and Linton (2012). ${ }^{3}$ Srisuma (2015) derived conditions for identification of parametrically specified per-period utilities by studying the pseudo-decision problem that underlies the two-step estimation approach for the DCDP model.

For the DCDP model with an unknown distribution of unobservables, Aguirregabiria (2010) studied identification of counterfactual choice probabilities for a finite horizon DCDP model assuming that the researcher observes a continuous outcome variable. Norets and Tang (2014) considered an infinite horizon DCDP model with discrete observed state variables. They showed that, when the distribution of unobservables is unknown, the per-period utilities and counterfactual choice probabilities are only set-identified even under parametric or shape restrictions on the per-period utilities. In this paper, we derive sufficient conditions for identification when continuous observed state variables are available. Our study is also related to a recent paper of Blevins (2014), who considered structural dynamic models that admit both discrete and continuous choices. When the joint distribution of shocks of the discrete choices is unknown, he adopted the exclusion restriction strategy and provided identification results in the setting where the agent's discrete action is in fact binary. The present paper focuses on the model where all choices are discrete and establishes identification for both binary and general multinomial choice settings with a nonparametric specification of the unobservables.

The rest of the paper is organized as follows. In Section 2, we present the setup and assumptions of the DCDP model. In Section 3, we discuss structural parameters of interest and characterize identification of the DCDP model. To motivate our identification strategy, we study identification of the model with an unknown distribution of unobservables in the simpler context where agents make binary 
choices in Section 4. In Section 5, we present identification results for the model with an unknown distribution of unobservables in the general multinomial choice setting. In Section 6, we discuss identification of extensions of the baseline model and conclude the paper. Appendix A provides proofs for all the theoretical results in the paper. Appendix B presents additional results on partial identification of the structural DCDP value functions when the data exhibit insufficient support.

\section{THE FRAMEWORK AND ASSUMPTIONS}

Time is discrete and indexed by $t \in\{1,2, \ldots\}$. Consider an economic agent with intertemporal utility that is additively separable over time. At each period, the agent makes a decision over $K$ mutually exclusive choices denoted as Choice $k, k \in C$, where $C \equiv\{1, \ldots, K\}$. Let $\left(x_{t}, \varepsilon_{t}\right)$ be the agent's state variables at time $t$ where the econometrician only observes $x_{t}$. The vector $\varepsilon_{t} \equiv\left(\varepsilon_{k, t}\right)_{k \in C}$ is unobserved, where $\varepsilon_{k, t}$ denotes the preference shock of Choice $k$.

At the beginning of each period $t,\left(x_{t}, \varepsilon_{t}\right)$ is revealed to the agent who then chooses an action $d_{t} \in C$ and receives the instantaneous return $u_{t}\left(d_{t}, x_{t}, \varepsilon_{t}\right)$. The next period state variables $\left(x_{t+1}, \varepsilon_{t+1}\right)$ remain uncertain to the agent. We assume that the transition of state variables follows a controlled first-order Markov process with transition probability density $f\left(x_{t+1}, \varepsilon_{t+1} \mid x_{t}, \varepsilon_{t}, d_{t}\right)$. Following Magnac and Thesmar (2002), we assume that agents' expectations are perfect in the sense that their beliefs about the evolution of the state variables coincide with that density. To proceed, as in Rust (1994) and Magnac and Thesmar (2002, pp. 802-804), we make the following assumptions on the agent's preference and on the laws of motion of the state variables.

[M1] (Additive Separability) For $k \in C$ and for all $t$,

$u_{t}\left(k, x_{t}, \varepsilon_{t}\right)=u_{k, t}^{*}\left(x_{t}\right)+\varepsilon_{k, t}$.

[M2] (Conditional Independence) For all $t$,

$f\left(x_{t+1}, \varepsilon_{t+1} \mid x_{t}, \varepsilon_{t}, d_{t}\right)=f_{\varepsilon}\left(\varepsilon_{t+1}\right) f_{x}\left(x_{t+1} \mid x_{t}, d_{t}\right)$.

Assumption [M1] is a standard assumption used in static random utility discrete choice models. [M2] assumes that the unobservables $\varepsilon_{t}$ are i.i.d. exogenous random shocks, while future values of the observed states $x_{t+1}$ can depend on the current control variable $d_{t}$ and values of the observed states $x_{t}$ but they do not directly depend on values of the current unobserved shock $\varepsilon_{t}$.

Let $\beta \in[0,1)$ be the discount factor. Assume that choices at each period are made to maximize the agent's discounted expected lifetime utility. By [M1], [M2] and perfect expectations, the value functions $\left(v_{k, t}\right)_{k \in C}$ of this model can be characterized by the following Bellman equations

$v_{k, t}\left(x_{t}, \varepsilon_{t}\right)=u_{k, t}^{*}\left(x_{t}\right)+\varepsilon_{k, t}+\beta E\left[\max _{j \in C}\left\{v_{j, t+1}\left(x_{t+1}, \varepsilon_{t+1}\right)\right\} \mid x_{t}, d_{t}=k\right]$. 
Therefore, for $k \in C$ and for all $t$, there is a value $v_{k, t}^{*}\left(x_{t}\right)$, which is a function of $x_{t}$ only, such that the choicewise value functions $v_{k, t}\left(x_{t}, \varepsilon_{t}\right)$ can be written as

$v_{k, t}\left(x_{t}, \varepsilon_{t}\right)=v_{k, t}^{*}\left(x_{t}\right)+\varepsilon_{k, t}$,

where $v_{k, t}^{*}\left(x_{t}\right)$ satisfies

$v_{k, t}^{*}\left(x_{t}\right)=u_{k, t}^{*}\left(x_{t}\right)+\beta E\left[\max _{j \in C}\left\{v_{j, t+1}^{*}\left(x_{t+1}\right)+\varepsilon_{j, t+1}\right\} \mid x_{t}, d_{t}=k\right]$.

The observed time series of the agent's choices is the sequence of optimal decisions $\left\{\delta_{t}\right\}$ satisfying

$\delta_{t}=\arg \max _{k \in C}\left\{v_{k, t}\left(x_{t}, \varepsilon_{t}\right)\right\}$.

In this paper, we study stationary infinite horizon models so that there is no terminal period to be exploited as a source of identification. We assume that the time horizon is infinite and that the per-period utilities and transition probabilities are time-invariant. The stationary Markovian structure of the model implies that the agent faces the same decision problem in state $\left(x_{s}, \varepsilon_{s}\right)$ at period $s$ and in state $\left(x_{t}, \varepsilon_{t}\right)$ at period $t$, provided that $\left(x_{s}, \varepsilon_{s}\right)=\left(x_{t}, \varepsilon_{t}\right)$. Hence we can suppress the time index and, for the rest of this paper, we denote variables $y$ and $y^{\prime}$ as the current and next period objects, respectively. Under this notation, we can formulate the value and policy functions of the DCDP model as follows.

$v_{k}^{*}(x)=u_{k}^{*}(x)+\beta E\left[\max _{j \in C}\left\{v_{j}^{*}\left(x^{\prime}\right)+\varepsilon_{j}^{\prime}\right\} \mid x, d=k\right]$ for $k \in C$,

$\delta=\arg \max _{k \in C}\left\{v_{k}^{*}(x)+\varepsilon_{k}\right\}$.

Assume the following:

[M3] For $k \in C, E\left(\left|\varepsilon_{k}\right|\right)<\infty$. Furthermore, $E\left(\varepsilon_{K}\right)=0$.

Assumption [M3] imposes location normalization for the random shock of one of the choices. ${ }^{4}$ We now describe the space of the agent's preference functions. Let $\Gamma$ be the Bellman operator that maps a $K$-dimensional vector of functions $h=\left(h_{k}\right)_{k \in C}$ to another $K$-dimensional vector of functions $\left(\Gamma_{k}(h)\right)_{k \in C}$ where, for $k \in C$,

$\Gamma_{k}(h)(x) \equiv u_{k}^{*}(x)+\beta E\left[\max _{j \in C}\left\{h_{j}\left(x^{\prime}\right)+\varepsilon_{j}^{\prime}\right\} \mid x, d=k\right]$.

Let $B$ be a Banach space of vectors of functions. Rust (1988) considered the case of $\left(u_{k}^{*}\right)_{k \in C} \in B$ where $B$ is the space of vectors of bounded functions. He established that there is a unique vector of functions $v^{*} \equiv\left(v_{k}^{*}\right)_{k \in C}$ that satisfies $v^{*}=\Gamma\left(v^{*}\right)$. In this paper, we allow for unbounded instantaneous utilities. Note that, for unbounded utilities $u_{k}^{*}$, the well-known Blackwell sufficient condition for 
contraction of $\Gamma$ (see, e.g., Stokey and Lucas (1989, Theorem 3.3)) is not directly applicable. To secure a unique solution to the system of Bellman equations (2.1), we adopt the weighted sup norm approach of Lippman (1975) and Norets (2010) as follows.

Let $q$ be a positive integer and $\alpha(x)$ be a function such that $1 \leq \alpha(x)<\infty$ for all $x$. Assume that there is a positive real constant $b$ such that for all $x$,

$\max _{k \in C} E\left(\alpha\left(x^{\prime}\right)^{n} \mid x, d=k\right) \leq(\alpha(x)+b)^{n}$ for $n \in\{1, \ldots, q\}$.

For any $K$-dimensional vector of functions $h$, we define the $\alpha$-weighted sup norm of $h$ as

$\|h\|_{\alpha} \equiv \sup _{x} \frac{\max _{k \in C}\left|h_{k}(x)\right|}{\alpha(x)^{q}}$.

We impose the following restriction on the agent's preference functions.

[M4] For some function $\alpha(x)$ and positive integer $q$, both $\left(u_{k}^{*}\right)_{k \in C}$ and $\left(v_{k}^{*}\right)_{k \in C}$ are elements of $B(\alpha, q)$, where $B(\alpha, q)$ denotes the space of $K$-dimensional vectors of functions bounded under the norm $\|\cdot\|_{\alpha}$.

Assumption [M4] allows for sup norm unbounded utilities but their growing rate must be regulated relatively to $\alpha(x)$, which itself may take a large value at the next period state but such an event should occur with small probability. The following lemma establishes that the vector of functions $\left(v_{k}^{*}\right)_{k \in C}$ can be solved as the unique fixed point of the Bellman operator $\Gamma$ and thus is the unique solution to the Bellman equations (2.1).

LEMMA 1. Given [M2], [M3] and [M4], the following statements hold: (i) If $h \in B(\alpha, q)$, then $\Gamma(h) \in B(\alpha, q)$. (ii) There is a unique vector of functions $v^{*}=\left(v_{1}^{*}, \ldots, v_{K}^{*}\right) \in B(\alpha, q)$ satisfying $v^{*}=\Gamma\left(v^{*}\right)$.

To proceed, we require further assumptions on the joint distribution of $\left(\varepsilon_{k}\right)_{k \in C}$.

[M5] The joint distribution of $\left(\varepsilon_{k}\right)_{k \in C}$ is absolutely continuous with respect to Lebesgue measure and has a joint density that is everywhere positive on $R^{K}$.

For any $K$-dimensional real vector $c$, let $\left(c_{k}-c_{j}\right)_{j \in C \backslash\{k\}}$ denote the $(K-1)$ dimensional vector $\left(c_{k}-c_{1}, \ldots, c_{k}-c_{K}\right)$ with the term $\left(c_{k}-c_{k}\right)$ excluded. Let $G_{k}$ denote the joint distribution of $-\left(\varepsilon_{k}-\varepsilon_{j}\right)_{j \in C \backslash\{k\}}$. Assumption [M5] ensures that the agent's optimal decision is almost surely unique. Moreover, it implies that for $k \in C$, the distribution $G_{k}$ is also absolutely continuous and has a Lebesgue density that is everywhere positive on $R^{K-1}$. Given [M2] and (2.2), the conditional choice probabilities of the DCDP model can be characterized as follows. For $k \in C$,

$$
P(\delta=k \mid x)=G_{k}\left(\left(v_{k}^{*}(x)-v_{j}^{*}(x)\right)_{j \in C \backslash\{k\}}\right) .
$$




\section{PARAMETERS OF INTEREST AND IDENTIFICATION}

We have a sample of individuals who follow the constituted DCDP model. The data consist of observed state variables and optimal decisions for all individuals and for at least two consecutive periods. Assuming random sampling, we can suppress the individual index. The parameters of interest in the DCDP model are $\left(u_{k}^{*}(x), v_{k}^{*}(x), \beta, G_{k}, f_{x}\left(x^{\prime} \mid x, d=k\right)\right)$ for $k \in C$.

Under perfect expectations and assumption [M2], the agent's belief about the transition of the observed states coincides with the density $f_{x}\left(x^{\prime} \mid x, d=k\right)$, which therefore equals the density of $x^{\prime}$ conditional on $x$ and $\delta=k$. Hence it can be nonparametrically identified using the data $\left(\delta, x, x^{\prime}\right)$. This fact has been used in the DCDP literature with a nonparametric specification of the transition law of the observed state variables (e.g., Magnac and Thesmar (2002), Bajari et al. (2009), and Srisuma and Linton(2012)). Therefore, we can replace the agent's control variable $d$ with the observed choice $\delta$ in the conditional expectation expressions of (2.1), (2.3), and (2.4).

Given $G_{K}$, we can deduce $G_{k}$ for all $k \in C \backslash\{K\}$ since $\left(\varepsilon_{j}-\varepsilon_{k}\right)_{j \in C \backslash\{k\}}$ can be expressed as a known linear transformation of $\left(\varepsilon_{j}-\varepsilon_{K}\right)_{j \in C \backslash\{K\}}$ (see Thompson (1989) and Matzkin (1993)). For notational simplicity, let $G$ denote the joint distribution $G_{K}$. Thus, it suffices to know $G$ to determine $G_{k}$ for all $k \in C \backslash\{K\}$.

Using conditions [M2] and [M3], we can further rewrite the systematic Bellman equations (2.1) as

$$
\begin{aligned}
& v_{k}^{*}(x)=u_{k}^{*}(x)+\beta E\left(v_{K}^{*}\left(x^{\prime}\right) \mid x, \delta=k\right) \\
& \quad+\beta E\left[\max _{j \in C}\left\{\Delta v_{j}^{*}\left(x^{\prime}\right)+\Delta \varepsilon_{j}^{\prime}\right\} \mid x, \delta=k\right],
\end{aligned}
$$

where $\Delta v_{j}^{*} \equiv v_{j}^{*}-v_{K}^{*}$ and $\Delta \varepsilon_{j} \equiv \varepsilon_{j}-\varepsilon_{K}$ for $j \in C$. We refer to $v_{k}^{*}$ as the derived structural parameters because, given the primitive structural parameters $\left(u_{k}^{*}(x), \beta, G\right)$, they can be deduced as the unique solution to the equation system (3.1). In line with the literature (e.g., Aguirregabiria and Mira (2002), Srisuma and Linton (2012), Norets and Tang (2014), and Srisuma (2015)), we assume throughout this paper that the researcher knows the discount factor $\beta .^{5}$ Thus we focus on identification of the remaining structural parameters $G$ and $\left(u_{k}^{*}(x), v_{k}^{*}(x)\right)$ for $k \in C$.

We now characterize identification for the DCDP model.

THEOREM 1. Given $\beta, u_{K}^{*}$ and $G$, the remaining structural parameters $\left(u_{1}^{*}, \ldots, u_{K-1}^{*}\right)$ and $\left(v_{1}^{*}, \ldots, v_{K}^{*}\right)$ are identified.

Magnac and Thesmar (2002, Proposition 2 (i)) characterize identification of the finite horizon DCDP model. Theorem 1 extends their results to the infinite horizon case allowing for unbounded per-period utilities. In Magnac and Thesmar's (2002) identification analysis, all the observed state variables $x$ are assumed to take only finite values. As noted by Manski(1988), a continuous 
distribution of unobservables cannot be nonparametrically identified with only discrete-valued regressors. There is scope for further relaxing the assumption on $G$ if the model admits continuous observed state variables.

However, when $G$ is unknown, following the arguments of Magnac and Thesmar (2002, Proposition 2 (ii)), we note that, for two distributions $G$ and $\widetilde{G}$, the configurations $\left(\beta, u_{K}^{*}, G\right)$ and $\left(\beta, u_{K}^{*}, \widetilde{G}\right)$ are observationally equivalent since, by Theorem 1 , the implied vectors of the remaining structural parameters derived from both configurations are compatible with the Bellman equations (3.1) and the observed conditional choice probabilities. Such observational equivalence holds regardless of continuity of $x$. Thus extra information on the modeling assumptions has to be supplied in lieu of $G$ to ensure identification. In the subsequent sections, we will provide sufficient conditions for identification of $G$ using restrictions on utility primitives and continuous observables by which we can proceed to identify the remaining structural parameters using the results of Theorem 1.

\section{IDENTIFICATION WHEN $G$ IS UNKNOWN IN THE BINARY CHOICE SETTING}

In this section, we analyze identification of the model with nonparametric unobservables within the binary choice context. This allows us to present the identifying strategy with simplified notation.

Let $C=\{1,2\}$. Hence $K=2$ and $G$ is the distribution function of $\left(\varepsilon_{1}-\varepsilon_{2}\right)$. We focus on the conditional choice probability equation

$P(\delta=2 \mid x)=G\left(v_{2}^{*}(x)-v_{1}^{*}(x)\right)$.

When $G$ is nonparametric, non-identification may arise due to composition between the unknown link function $G$ and the unknown index function $v_{2}^{*}-v_{1}^{*}$ in equation (4.1). By (3.1),

$v_{2}^{*}(x)-v_{1}^{*}(x)=u_{2}^{*}(x)+\lambda(x)$,

where

$$
\begin{aligned}
\lambda(x) \equiv & m(x)-u_{1}^{*}(x), \\
m(x) \equiv & \beta\left[E\left(v_{2}^{*}\left(x^{\prime}\right) \mid x, \delta=2\right)-E\left(v_{2}^{*}\left(x^{\prime}\right) \mid x, \delta=1\right)\right] \\
& +\beta E\left[\max _{j \in C}\left\{\Delta v_{j}^{*}\left(x^{\prime}\right)+\Delta \varepsilon_{j}^{\prime}\right\} \mid x, \delta=2\right] \\
& -\beta E\left[\max _{j \in C}\left\{\Delta v_{j}^{*}\left(x^{\prime}\right)+\Delta \varepsilon_{j}^{\prime}\right\} \mid x, \delta=1\right] .
\end{aligned}
$$

Note that $m(x)$ represents the difference between the discounted expected future values for making Choice 2 and for making Choice 1 at the current period. This difference is zero if future state variables are (conditionally) independent of the current action. In general, when $G$ is unknown, $m(x)$ is an unknown non-trivial function of $x$. 
To overcome the identification problem, given $\beta$ and $u_{2}^{*}$, the effects of $v_{2}^{*}-v_{1}^{*}$ and $G$ on the conditional choice probability can be disentangled if we can fix $\lambda(x)$ but simultaneously freely move $u_{2}^{*}$. In this case, we can trace out the distribution $G$ by varying $u_{2}^{*}$, and thus $G$ can be identified. Such a variation-free condition does not hold if $\lambda(x)$ and $u_{2}^{*}(x)$ share the exact same set of observed state variables. Therefore, to achieve identification, we propose imposing an exclusion restriction to provide such a source of variation. Theorem 2 demonstrates this identification strategy.

Decompose the vector $x$ as $x=(w, z)$ where the subvectors $w$ and $z$ have no common component and are non-empty. Denote the support of $x$ as $\Gamma_{X}=\Gamma_{W} \times$ $\Gamma_{Z}$, where $\Gamma_{W}$ and $\Gamma_{Z}$ are the supports of $w$ and $z$, respectively.

THEOREM 2. Let $u_{2}^{*}(x)=u_{2}^{*}(w, z)$ be a known function. Assume also the following: (i) $u_{1}^{*}(x)$ is a function of $w$ only. (ii) $\left(w^{\prime}, z^{\prime}\right) \perp z \mid w, \delta$. (iii) The $\tau$-quantile of $\varepsilon_{1}-\varepsilon_{2}$ is zero for some known $\tau \in(0,1)$. (iv) For each $w \in \Gamma_{W}$, the distribution of $u_{2}^{*}(w, z)$ conditional on $w$ has a Lebesgue density that is everywhere positive on the real line. Then both $G$ and $\lambda$ are identified.

Assumption (i) may be justified if there are choice-specific attributes. This assumption does not preclude common attributes since the vector $w$ is allowed to appear in both $u_{1}^{*}$ and $u_{2}^{*}$. However, assumption (i) alone is not sufficient to yield the required exclusion restriction since the choice-specific attributes may enter both value functions via the information set that the agent uses to form their expected future values. Assumption (ii) is sufficient to remove such an effect by regulating the predictability of these attributes through a conditional independence assumption. In fact, assumption (ii) essentially requires that, conditional on $\delta, w$ serves as a sufficient statistic for predicting all observed state variables of the next period. A sufficient condition to validate assumption (ii) is that the transition density $f_{w, z}\left(w^{\prime}, z^{\prime} \mid w, z, \delta\right)$ satisfies

\section{Assumption (ii*)}

$f_{w, z}\left(w^{\prime}, z^{\prime} \mid w, z, \delta\right)=f_{z}\left(z^{\prime} \mid w^{\prime}, \delta\right) f_{w}\left(w^{\prime} \mid w, \delta\right)$.

Assumption (ii*) implies that $z^{\prime} \perp(w, z) \mid w^{\prime}, \delta$ and $w^{\prime} \perp z \mid w, \delta$. Hence, the influence of the current state $z$ on the next period states $\left(w^{\prime}, z^{\prime}\right)$ can mainly be enacted through the current choice $\delta$. In other words, $z$ is necessarily not a purely persistent state variable and cannot directly affect the evolution of the time series process of $w$. See Figure 1 for a graphical illustration of the transition of the observables under assumption (ii*).

We now discuss the remaining assumptions of Theorem 2 as follows. Because the function $\lambda$ is unknown and the condition $u_{2}^{*}(x)+\lambda(x) \geq \varepsilon_{1}-\varepsilon_{2}$ is invariant with respect to an arbitrary constant added to both sides of the inequality, we can only identify $G$ up to a location normalization, which is imposed by assumption (iii) of the theorem. Finally, assumption (iv) implies that $z$ cannot be a proper 


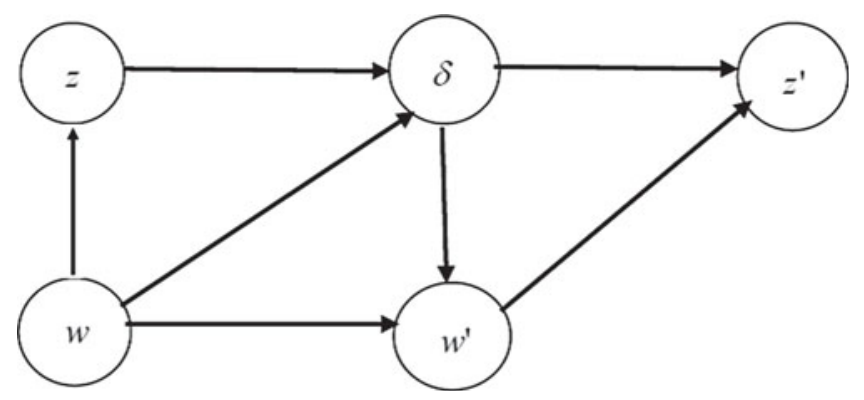

FIGURE 1. Transition of the observables under assumption (ii*).

subvector of $w$ and furthermore $u_{2}^{*}(w, z)$ as a random variable should be continuous and have large support. The large support assumption is used to trace out $G$ over its entire domain. This assumption could be a good approximation to the situation where $u_{2}^{*}(w, z)$ exhibits a large range of variation in the data and there is no natural prior bound on its variation. The large support condition is also commonly imposed for identification of random utility discrete choice models with nonparametric unobservables (e.g., Manski (1985), Horowitz (1992, 1993), Matzkin (1992, 1993), Lewbel (2000, 2014), and de Jong and Woutersen (2011)). We can relax this condition but the distribution $G$ may only be partially identified. See Section 4.1 for further details.

We further comment on our identification strategy. Under [M2] and assumptions (i) and (ii) of Theorem $2, \lambda(x)$ is some function $\bar{\lambda}(w)$ of $w$ only. Hence, given (4.1) and (4.2), we have

$$
P(\delta=2 \mid w, z)=G\left(u_{2}^{*}(w, z)+\bar{\lambda}(w)\right) .
$$

Because $u_{2}^{*}$ is known and depends on the excluded variable $z$, equation (4.5) indicates that we can fix the states $w$ and vary the value of $z$ so as to trace out the distribution $G$. Therefore, assumptions (i) and (ii) are critical to form the exclusion restriction for identification of $G$. We note that these two identifying conditions are imposed on the shape of the structural primitive functions. Assumption (i) imposes shape restrictions on the primitive utility $u_{1}^{*}$. This assumption alone is not testable and may be part of the researcher's belief in modeling a particular application. ${ }^{6}$ On the other hand, assumption (ii) is directly testable since its restrictions are on the joint time series of the observed state variables.

The identification result of Theorem 2 can be achieved using only one excluded variable $z$. Over-identification may thus arise if more than one exclusion restriction is available. Note that Theorem 2 assumes that the per-period utility $u_{2}^{*}$ is known a priori. This assumption can be further relaxed when the model admits more than one excluded variable. Let $z=\left(z_{1}, \ldots, z_{J}\right)$ be a $J$-dimensional vector of excluded variables. Suppose that $u_{2}^{*}(x)$ is specified up to a $J$-dimensional vector of parameters $\theta$. The next theorem establishes identification of $\theta$ when the utility $u_{2}^{*}$ is specified as linear in parameters. 
THEOREM 3. Suppose assumptions (i) and (ii) of Theorem 2 hold and that $u_{2}^{*}(x)=\theta^{\prime} h(w, z)$ where $\left|\theta_{1}\right|=1$ and $h(w, z)=\left(h_{1}\left(w, z_{1}\right), \ldots, h_{J}\left(w, z_{J}\right)\right)$ is a $J$-dimensional vector of known functions. Let $\partial h_{j}\left(w, z_{j}\right) / \partial z_{j}$ denote the partial derivative of $h_{j}\left(w, z_{j}\right)$ with respect to $z_{j}$. Assume that for all $j \in\{1, \ldots, J\}$, $\partial h_{j}\left(w, z_{j}\right) / \partial z_{j}$ is non-zero with probability 1 . Then the vector $\theta$ is identified.

When $\theta, \lambda$ and $G$ are unknown, the vector $\theta$ is identified up to a scale normalization since the condition $\theta^{\prime} h(w, z)+\lambda(x) \geq \varepsilon_{1}-\varepsilon_{2}$ for making Choice 2 is invariant with respect to an arbitrary positive scalar used to multiply both sides of the inequality. Theorem 3 normalizes the scale by imposing a unity value for the magnitude of $\theta_{1}$. Using $u_{2}^{*}(x)=\theta^{\prime} h(w, z)$ in equation (4.5), we have

$P(\delta=2 \mid w, z)=G\left(\theta^{\prime} h(w, z)+\bar{\lambda}(w)\right)$.

Since both $h_{j}\left(w, z_{j}\right)$ and $\bar{\lambda}(w)$ of equation (4.6) depend on the variables $w$, the excluded variables $z_{j}$ can thus be interpreted as the identifying "instrumental variables" in the sense that they can vary the observed object $h_{j}\left(w, z_{j}\right)$ (the "rank" condition) but cannot affect the "unobserved" object $\bar{\lambda}(w)$ (the "exclusion" condition).

It is noted that identification of $\theta$ in Theorem 3 requires continuous excluded variables $z$ but this result does not require a large support assumption on any of the components $h_{j}\left(w, z_{j}\right)$ of the utility $u_{2}^{*}(w, z)$. Once the vector of utility parameters $\theta$ is identified, we can proceed to identify $G$ by applying the results of Theorem 2 . Then, given $\left(\beta, u_{2}^{*}, G\right)$ and by application of Theorem 1 , the remaining structural functions $\left(u_{1}^{*}, v_{1}^{*}, v_{2}^{*}\right)$ of this model are also identified.

\subsection{Partial identification under limited support of the observables}

Assumption (iv) of Theorem 2 imposes that $u_{2}^{*}(w, z)$ should have a rich support. Such a condition seems difficult to avoid for the purpose of point identification of the DCDP structural parameters. When the data lack sufficient support, the distribution $G(t)$ may be only identified over those $t$ values about which the observables are informative. Relaxing the large support assumption, we now derive identification of $G$ in the next theorem, which generalizes Theorem 2 of the paper.

THEOREM 4. Suppose that all assumptions of Theorem 2 hold, except that assumption (iv) is replaced by the following assumption: (iv*) For each $w \in \Gamma_{W}$, there is an open interval containing zero such that the distribution of $u_{2}^{*}(x)+$ $\lambda(x)$ conditional on $w$ has a Lebesgue density that is everywhere positive on this interval. Then the function $\lambda$ is identified. Moreover, let $\Omega_{12}$ denote the support of $v_{2}^{*}(x)-v_{1}^{*}(x)$. Then the function $G(t)$ is identified at every $t \in \Omega_{12}$.

Assumption (iv*) imposes a much weaker support condition than assumption (iv) of Theorem 2. In fact, assumption (iv) is sufficient for assumption (iv*) and implies that $\Omega_{12}$ is the entire real line by which $G(t)$ is identified at each $t \in R$. 
Using equation (4.5), we can deduce

$P\left(\delta=2 \mid u_{2}^{*}(w, z), w\right)=G\left(u_{2}^{*}(w, z)+\bar{\lambda}(w)\right)$.

Consider the event that the expression $P\left(\delta=2 \mid u_{2}^{*}(w, z), w\right)$ takes a value in an open interval having radius $\eta$ and containing $\tau$. Given assumption (iii) of Theorem 2, assumption (iv*) is fulfilled provided that, conditional on $w$ and for all $\eta>0$, the probability of this event is strictly positive. By continuity and monotonicity of $G$, assumption (iv*) guarantees that, for each $w \in \Gamma_{W}$, there is some $z \in \Gamma_{Z}$ such that $P(\delta=2 \mid w, z)=\tau$. Hence given assumptions (i), (ii), and (iii) of Theorem 2 and knowledge of $u_{2}^{*}$, assumption (iv*) is empirically verifiable even though its statement concerns the unknown function $\lambda$.

When the support of $v_{2}^{*}(x)-v_{1}^{*}(x)$ is not rich enough to cover the entire domain of $G$, the distribution function $G(t)$ may not be identified at every point in its domain but it remains identified at each $t$ in the support of the value difference. Hence, lack of adequate support may limit the usefulness of the exclusion restrictions of this paper for nonparametrically point identifying the structural primitives and the resulting counterfactuals. Nevertheless, partial identification of $G$ still enables us to separately bound the value functions $v_{1}^{*}$ and $v_{2}^{*}$. See Appendix B of the present paper for the derivation of these bounds. The partial identification results still provide implicit restrictions on the set of observationally equivalent structural primitives and hence remain useful for evaluating distributional assumptions for modeling the unobservables.

\subsection{An example : optimal replacement of production capital}

To illustrate the use of our identification method, we consider the following simple version of the machine replacement decision model in the empirical DCDP literature (e.g., Rust (1987), Kennet (1994), and Cho (2011)).

Consider a firm with multiple production assets (machines). At every period, the firm manager has to decide whether an existing machine should be replaced with a new one. Let Choice 1 be the decision of continuing to operate the old machine and Choice 2 be that of replacement with a completely new one. Following Rust (1987), we assume that the firm manager behaves as a cost minimizer with instantaneous choicewise utilities

$u_{1}\left(x, \varepsilon_{1}\right)=-c(y)+\varepsilon_{1}$,

$u_{2}\left(x, \varepsilon_{1}\right)=z-p+\varepsilon_{2}$,

where $y$ is a vector of observed states capturing the operational history of the machine such as the cumulative usage of the machine and the experienced number of shutdowns since the last replacement, $z$ is the manager's booked (estimated) machine net scrap value, $p$ is the market price of a new machine, $\varepsilon_{1}$ can be interpreted as the unobserved cost of operating an old machine, and $\varepsilon_{2}$ can account for both the unobserved operating cost of a new machine and any discrepancy between $z$ 
and the actual scrap value of the old machine. In this model, $u_{1}^{*}(x)=-c(y)$ and $u_{2}^{*}(x)=z-p$, where $x=(w, z)$ and $w=(y, p)$. The parameters of interest are the maintenance cost function $c(y)$ and the value functions $v_{1}^{*}$ and $v_{2}^{*}$.

We verify key assumptions of Theorem 2 for this example. Assumption (i) holds under specification (4.8) and requires that the maintenance cost should not depend on the scrap value $z$. Assumption (ii) concerns the law of motion of the observables. As discussed above, this assumption is imposed on the observed state variables and hence is empirically testable. Since the price process is generally serially correlated, its evolution may violate assumption (ii). On the other hand, $z$ may be a potential candidate for a valid excluded variable that satisfies both assumptions (i) and (ii). We can justify assumption (ii) by verifying assumption (ii*), which suffices for the required conditional independence condition. Assumption (ii*) may be plausible when the manager estimates the machine scrap value based on its usage information $y$ and the market price $p$ plus some independent random noise reflecting the manager's assessment of the current status of the machine. Note that assumption (ii*) also precludes the case that the scrap value $z$ may directly affect the evolution of both the usage $y$ and the price $p$.

We now discuss the other identifying assumptions. Assumption (iii) is a location normalization condition, which holds if we specify that the median of $\varepsilon_{1}-\varepsilon_{2}$ is zero. Assumption (iv) requires that $z$ should exhibit large variation conditional on $(y, p)$. If all these conditions are fulfilled, we can identify the distribution of $\varepsilon_{1}-\varepsilon_{2}$ by applying Theorem 2 . Then we identify the cost function $c(y)$ and the value functions $v_{1}^{*}$ and $v_{2}^{*}$ by using the identification results of Theorem 1 . When the large support condition of assumption (iv) does not hold, we can use assumptions (iv*) of Theorem 4 instead. Given (4.8), (4.9), assumption (ii) and the zero median restriction, equation (4.5) becomes

$P(\delta=2 \mid y, p, z)=G(z-p+\bar{\lambda}(y, p))$

and assumption (iv*) thus holds provided that, conditional on $(y, p)$, for all $\eta>0$, the set of $z$ values (depending on $\eta)$ for which $P(\delta=2 \mid y, p, z)$ takes a value in an open interval having radius $\eta$ and containing 0.5 occurs with positive probability. Clearly, this condition is empirically verifiable and may hold even if all the observed state variables have bounded support. Then, by Theorem 4, we can still partially identify the distribution of $\varepsilon_{1}-\varepsilon_{2}$ by which we may also construct bounds for the value functions $v_{1}^{*}$ and $v_{2}^{*}$ using the results developed in Appendix $\mathrm{B}$ of the paper.

Finally, we conclude this example by commenting on the observability of the variable $z$. In this example, $z$ may be a proxy of the manager's estimated scrap value of the machine. Such a proxy may be observed in the firm's financial statements. We note that the excluded variable $z$ is similar to the instrumental variable used in the estimation of endogenous reduced form models. If such a variable can be observed with sufficient variation, we may exploit its power to identify the DCDP structural parameters under nonparametric specification of the unobservables. 


\section{IDENTIFICATION WHEN $G$ IS UNKNOWN IN THE MULTINOMIAL CHOICE SETTING}

We now present our main identification results for the general multinomial choice dynamic programming model with nonparametric unobservables. Let $C \equiv\{1, \ldots, K\}$ be the choice set for any $K \geq 2$. We assume that both $\beta$ and $u_{K}^{*}$ are known and we seek conditions under which $G$, the joint distribution of $\left(\varepsilon_{j}-\varepsilon_{K}\right)_{j \in C \backslash\{K\}}$, is identified.

Using the analysis of Section 4 , for $j \in C \backslash\{K\}$, we write

$v_{K}^{*}(x)-v_{j}^{*}(x)=u_{K}^{*}(x)+\lambda_{j}(x)$

where

$$
\begin{aligned}
\lambda_{j}(x) \equiv & m_{j}(x)-u_{j}^{*}(x), \\
m_{j}(x) \equiv & \beta\left[E\left(v_{K}^{*}\left(x^{\prime}\right) \mid x, \delta=K\right)-E\left(v_{K}^{*}\left(x^{\prime}\right) \mid x, \delta=j\right)\right] \\
& +\beta E\left[\max _{k \in C}\left\{\Delta v_{k}^{*}\left(x^{\prime}\right)+\Delta \varepsilon_{k}^{\prime}\right\} \mid x, \delta=K\right] \\
& -\beta E\left[\max _{k \in C}\left\{\Delta v_{k}^{*}\left(x^{\prime}\right)+\Delta \varepsilon_{k}^{\prime}\right\} \mid x, \delta=j\right] .
\end{aligned}
$$

Decompose the vector $x$ as $x=(w, z)$, where $z=\left(z_{1}, \ldots, z_{K-1}\right)$ such that $w$ and the subvectors $z_{j}, j \in C \backslash\{K\}$ have no common component. Denote the support of $x$ as $\Gamma_{X}=\Gamma_{W} \times \Gamma_{Z}$, where $\Gamma_{W}$ and $\Gamma_{Z}$ denote the supports of $w$ and $z$, respectively. Recall that $G=G_{K}$ and, for $k \in C, G_{k}$ denotes the joint distribution of $\left(\varepsilon_{j}-\varepsilon_{k}\right)_{j \in C \backslash\{k\}}$. The joint distribution of $\left(\varepsilon_{j}\right)_{j \in C}$ remains nonparametric, but the following restriction is imposed for identification.

Condition 1. For any $K$-dimensional real vector $c$ and for any two indices $i, j \in C$,

$c_{i}>c_{j}$ if and only if $G_{i}\left(\left(c_{i}-c_{k}\right)_{k \in C \backslash\{i\}}\right)>G_{j}\left(\left(c_{j}-c_{k}\right)_{k \in C \backslash\{j\}}\right)$.

Let $z_{(j)}$ denote the subvector of $z$ excluding the variates $z_{j}$. Let $\Gamma_{Z_{(j)}}$ be the support of $z_{(j)}$. Note that $z_{(K-1)}$ is an empty vector when $K=2$. To simplify the exposition, if $K=2$, we adopt the interpretation that the pair $\left(w, z_{(K-1)}\right)$ reduces to $w$ and the statement $\left(w, z_{(K-1)}\right) \in \Gamma_{W} \times \Gamma_{Z_{(K-1)}}$ means $w \in \Gamma_{W}$.

The following theorem establishes identification of $G$ and $\lambda_{j}(x)$ for $j \in C \backslash\{K\}$.

THEOREM 5. Suppose Condition 1 holds. Assume that the following also hold: (i) $u_{1}^{*}(x)$ is a function of $w$ only. For $j \in\{2, \ldots, K\}, u_{j}^{*}(x) \equiv \bar{u}_{j}^{*}\left(w, z_{j-1}\right)$ is a function of $\left(w, z_{j-1}\right)$ only. The function $\bar{u}_{K}^{*}\left(w, z_{K-1}\right)$ is known. (ii) $\left(w^{\prime}, z^{\prime}\right) \perp$ $z \mid w, \delta$. (iii) For all $\left(w, z_{(K-1)}\right) \in \Gamma_{W} \times \Gamma_{\left.Z_{(K-1}\right)}$, the distribution of $\bar{u}_{K}^{*}\left(w, z_{K-1}\right)$ conditional on $\left(w, z_{(K-1)}\right)$ has a Lebesgue density that is everywhere positive on the real line. (iv) There is a value $c$ in $\Gamma_{W}$ such that the joint distribution of 
$\left(u_{j}^{*}(x)\right)_{j \in\{2, \ldots, K\}}$ conditional on $w=c$ has a Lebesgue density that is everywhere positive on $R^{K-1}$. Then the functions $G$ and $\lambda_{j}, j \in C \backslash\{K\}$ are identified.

Assumptions (i) and (ii) of Theorem 5 are analogous to those of Theorem 2. Note that, in the setting with $K$ choices, Theorem 5 requires at least $K-1$ exclusion restrictions, thus indicating the presence of a curse of dimensionality for identification of the multinomial choice model with nonparametric unobservables. This can also be seen from equation (2.6) expressing the conditional choice probability as a multivariate link function with $K-1$ indices. To identify the unknown link function, we need to freely vary all these indices. Hence, the required number of identifying exclusion restrictions increases with the number of available choices. Assumption (iii) of Theorem 5 is the multinomial counterpart of assumption (iv) of Theorem 2 and can therefore be interpreted in a similar fashion. Assumption (iv) of Theorem 5 enables the $K-1$ indexing functions in equation (2.6) to freely vary over a wide range such that, given these indices, we can trace out the value of $G$ at every point of its domain.

We now discuss the identifying power delivered by Condition 1. By equation (2.6), Condition 1 essentially states that the agent's systematic choicewise values rank order the conditional choice probabilities. For $K=2$, this condition is equivalent to assumption (iii) of Theorem 2 with the quantile $\tau$ specified to be 0.5 . Hence, in the binary choice setting, Theorem 5 reduces to Theorem 2 with the zero median restriction being imposed for location normalization of $G$. In the multinomial choice context, Condition 1 allows for identification of the unknown value differences $v_{K}^{*}-v_{j}^{*}$ by comparing the relative order of the observed probabilities of making choices $K$ and $j$ with that of the choice probabilities implied by other structural parameter configurations.

Condition 1 restricts the class of joint distributions of $\left(\varepsilon_{j}\right)_{j \in C}$ but is still quite flexible. Manski (1975) pioneered the use of such assumptions which were also imposed later by Matzkin (1993) and Fox (2007) as identifying restrictions in semiparametric multinomial choice models. Condition 1 is also studied as a property of monotonicity in the literature on quantal response equilibrium (e.g., McKelvey and Palfrey (1995) and Goeree, Holt, and Palfrey (2005)). Goeree et al. (2005, Proposition 5) and Fox (2007) indicated that a sufficient primitive assumption for Condition 1 is that the joint density of $\left(\varepsilon_{j}\right)_{j \in C}$ possesses the interchangeability property. ${ }^{7}$ Interchangeability holds for i.i.d. choicewise shocks, thus including Rust's (1994) dynamic multinomial logit specification as a special case. Interchangeability also holds for non-independent random shocks (see, e.g., Fox (2007)). Note that interchangeability is merely sufficient and that Condition 1 can also hold for some non-interchangeable unobserved shocks (Goeree et al. (2005)).

As in the binary choice case, we can relax the assumption that the utility $u_{K}^{*}(x)$ is known a priori if $z_{K-1}$, the vector of Choice $K$-specific variables, may contain more than one component. Let $z_{K-1}=\left(z_{1, K-1}, \ldots, z_{J, K-1}\right)$ be a $J$-dimensional vector of excluded variables. With $u_{K}^{*}$ specified as linear in a vector of parameters 
$\theta$, the next theorem establishes identification of $\theta$, thus generalizing the identification result of Theorem 3 .

THEOREM 6. Suppose assumptions (i) and (ii) of Theorem 5 hold and that $u_{K}^{*}(x)=\theta^{\prime} h\left(w, z_{K-1}\right)$ where $\left|\theta_{1}\right|=1$ and $h\left(w, z_{K-1}\right)=$ $\left(h_{1}\left(w, z_{1, K-1}\right), \ldots, h_{J}\left(w, z_{J}, K-1\right)\right)$ is a J-dimensional vector of known functions. Let $\partial h_{j}\left(w, z_{j, K-1}\right) / \partial z_{j, K-1}$ denote the partial derivative of $h_{j}\left(w, z_{j, K-1}\right)$ with respect to $z_{j, K-1}$. Assume that for all $j \in\{1, \ldots, J\}, \partial h_{j}\left(w, z_{j, K-1}\right) / \partial z_{j, K-1}$ is non-zero with probability 1. Then the vector $\theta$ is identified.

We now consider relaxing the large support assumptions (iii) and (iv) of Theorem 5. As in the binary choice case, we can also identify the distribution $G(t)$ for $t$ in the range of the value differences. The following theorem establishes this partial identification result for the multiple choice context.

THEOREM 7. Suppose Condition 1 and assumptions (i) and (ii) of Theorem 5 hold. Assume also the following: (iii*) For all $\left(w, z_{(K-1)}\right) \in \Gamma_{W} \times \Gamma_{Z_{(K-1)}}$ and for $j \in C \backslash\{K\}$, there is an open interval containing zero such that the distribution of $u_{K}^{*}(x)+\lambda_{j}(x)$ conditional on $\left(w, z_{(K-1)}\right)$ has a Lebesgue density that is everywhere positive on this interval. Then the functions $\lambda_{j}, j \in C \backslash\{K\}$ are identified. Moreover, let $\Omega_{K}$ denote the support of $\left(v_{K}^{*}(x)-v_{j}^{*}(x)\right)_{j \in C \backslash\{K\}}$. Then the function $G(t)$ is identified at every $t \in \Omega_{K}$.

\section{DISCUSSION AND CONCLUSION}

This paper develops new identification results for the class of discrete choice dynamic programming (DCDP) models within Rust's (1994) framework. We assume that the per-period utility of one of the choices is known but that the distribution of unobserved state variables, law of motion of observed state variables, and per-period utilities of all the other choices are nonparametrically specified.

We derive sufficient conditions for identification of the deep structural parameters of the DCDP model with nonparametric unobserved state variables. Our identification strategy does not rely on the use of terminal period data and hence can be applied to infinite horizon structural dynamic models. We assume that the agent's per-period utilities admit continuous observed choice-specific state variables that satisfy certain conditional independence conditions on the joint time series of observables. These conditions allow us to form exclusion restrictions to secure identification when information about distribution of the unobservables is not available.

We now discuss applications of our identification approach to extensions of the baseline DCDP model. Though Assumption [M2] is common in the DCDP literature, it requires that the unobservables should be independent of the intra-period observed states. We can relax this assumption to allow $G_{k}(\cdot \mid x)$, the joint distribution of $\left(\varepsilon_{j}-\varepsilon_{k}\right)_{j \in C \backslash\{k\}}$ conditional on $x$, to vary across $x$. By strengthening Condition 1 so that statement (5.4) holds with $G_{k}(\cdot \mid x)$ in place of $G_{k}(\cdot)$, the resulting 
DCDP model still admits the key identifying property that the agent's systematic choicewise values rank order the conditional choice probabilities. Hence, we can utilize assumptions (i), (ii), and (iii) of Theorem 5 to identify the value differences $\left(v_{k}^{*}(x)-v_{j}^{*}(x)\right)_{j \in C \backslash\{k\}}$. Provided that the excluded variables $z$ also satisfy the "special regressor" condition (see, e.g., Lewbel $(2000,2014)$ and Lewbel and Tang (2015)) that $\left(\varepsilon_{j}-\varepsilon_{K}\right)_{j \in C \backslash\{K\}} \perp z \mid w$, we can further trace out and thus identify $G_{k}(\cdot \mid x)=G_{k}(\cdot \mid w)$ by varying the observed states $z$.

Our method can also be extended to DCDP models with persistent unobserved heterogeneity. For such models, we can use the recent identification results of Kasahara and Shimotsu (2009) and Hu and Shum (2012) to nonparametrically identify the joint Markov transition law of the observed and the persistent unobserved state variables and also the choice probabilities conditional on these variables. With knowledge of these objects, we can then proceed to apply the identification strategy of this paper to the model augmented with persistent unobserved states.

We conclude the paper by remarking on the practical relevance of the identification results. In light of the identification analysis set forth in this paper, the researcher is encouraged to collect and explore data on the identifying excluded variables. Provided that such variables are observed with sufficient variation, the identification results can be utilized to implement an estimation procedure by which inference on structural primitives and the resulting counterfactual policy functionals can be more robust against misspecification of the unobservables. The present paper focuses on point identification of the DCDP structural parameters. This requires that variation of the value differences induced by the excluded variables should be no smaller than that of the differences of the unobservables. Lack of adequate variation may limit the usefulness of the excluded variables for the purpose of point identification. Nevertheless, the exclusion restriction can still yield identifying power that allows for set identification of the distribution of unobservables. The partial identification results established in this paper are also useful in their own right and can be further developed into a diagnostic device for parametric specifications of the DCDP model.

\section{NOTES}

1. See, e.g., Manski (1975, 1985), Klein and Spady (1993), Horowitz (1992, 1993), and Lewbel (2000) for estimation of static discrete choice models assuming parametric systematic utilities and nonparametric distribution of unobservables. Matzkin (1992,1993) and Lewbel and Linton(2007) consider nonparametric discrete choice models by further relaxing the parametric assumptions on the systematic utility to conditions on the utility function space defined under certain shape restrictions.

2. We also note that exclusion restrictions motivated by the underlying economic contexts can be useful for identification of agents' payoffs in discrete choice models with strategic interaction (see, e.g., Sanches, Silva-Junior, and Srisuma (2014) and Lewbel and Tang (2015)).

3. The two-step approach pioneered by Hotz and Miller(1993) is also widely used in the estimation of dynamic Markov games. For related literature, see, e.g., Aguirregabiria and Mira (2007), Bajari, Benkard, and Levin (2007), Pakes, Ostrovsky, and Berry (2007), Pesendorfer and SchmidtDengler (2008), Bajari, Chernozhukov, Hong, and Nekipelov (2009), and Sanches, Silva-Junior, and Srisuma (2013). 
4. As in static discrete choice models, the location of the marginal distribution of $\varepsilon_{k}$ is not separately identified since only the difference matters for the agent's choice behavior. Magnac and Thesmar (2002, p. 803) assume that $E\left(\varepsilon_{k}\right)$ is zero for all $k$. Such an assumption is stronger than that required.

5. Manski (1993) showed that one cannot distinguish between myopic $(\beta=0)$ and forward-looking $(\beta>0)$ agents if information on agents' utility primitives is not available.

6. Combined with assumption (ii) and knowledge of $u_{2}^{*}$, assumption (i) implies that $P(\delta=2 \mid w, z)$ is monotone in $z$ if and only if $u_{2}^{*}(w, z)$ is also monotone in $z$. Hence, subject to validity of the other identifying assumptions, we can form an empirically testable necessary (but not sufficient) condition for assumption (i).

7. See Goeree etal. (2005, p. 359) and Fox (2007, p. 1006) for a formal definition of the interchangeable joint density.

\section{REFERENCES}

Aguirregabiria, V. (2010) Another look at the identification of dynamic discrete decision processes: An application to retirement behavior. Journal of Business and Economic Statistics 28, 201-218.

Aguirregabiria, V. \& P. Mira (2002) Swapping the nested fixed point algorithm: A class of estimators for discrete Markov decision models. Econometrica 70, 1519-1543.

Aguirregabiria, V. \& P. Mira (2007) Sequential estimation of dynamic discrete games. Econometrica $75,1-53$.

Aguirregabiria, V. \& P. Mira (2010) Dynamic discrete choice structural models: A survey. Journal of Econometrics 156, 38-67.

Bajari, P., C.L. Benkard, \& J. Levin (2007) Estimating dynamic models of imperfect competition. Econometrica 75, 1331-1370.

Bajari, P., V. Chernozhukov, H. Hong, \& D. Nekipelov (2009) Nonparametric and Semiparametric Analysis of a Dynamic Discrete Game. Working paper, University of Minnesota.

Blevins, J. (2014) Nonparametric identification of dynamic decision processes with discrete and continuous choices. Quantitative Economics 5, 531-554.

Chen, L-Y. (2007) Semiparametric Identification of Structural Dynamic Optimal Stopping Time Models. Cemmap Working paper CWP06/07, Centre for Microdata Methods and Practice, Institute for Fiscal Studies.

Chen, L-Y. (2009) Identification of Structural Dynamic Discrete Choice Models. Cemmap Working paper CWP08/09, Centre for Microdata Methods and Practice, Institute for Fiscal Studies.

Cho, S-J. (2011) An empirical model of mainframe computer investment. Journal of Applied Econometrics 26, 122-150.

de Jong, R.M. \& T. Woutersen (2011) Dynamic time series binary choice. Econometric Theory 27, 673-702.

Denardo, E. (1967) Contraction mappings in the theory underlying dynamic programming. SIAM Review 9, 165-177.

Eckstein, Z. \& K. Wolpin (1989) The specification and estimation of dynamic stochastic discrete choice models: A survey. Journal of Human Resources 24, 562-598.

Fox, J.T. (2007) Semiparametric estimation of multinomial discrete-choice models using a subset of choices. RAND Journal of Economics 38, 1002-1019.

Goeree, J.K., Holt, C.A., \& Palfrey, T.R. (2005) Regular quantal response equilibrium. Experimental Economics 8, 347-367.

Heckman, J. \& S. Navarro (2007) Dynamic discrete choice and dynamic treatment effects. Journal of Econometrics 136, 341-396.

Horowitz, J. (1992) A maximum score estimator for the binary response model. Econometrica 60, 505-531.

Horowitz, J. (1993) Optimal rates of convergence of parameter estimators in the binary response model with weak distributional assumptions. Econometric Theory 9, 1-18. 
Hotz, V. \& R.A. Miller (1993) Conditional choice probabilities and the estimation of dynamic models. Review of Economic Studies 60, 497-531.

Hotz, V., R.A. Miller, S. Sanders, \& J. Smith (1994) A simulation estimator for dynamic models of discrete choice. Review of Economic Studies 61, 265-289.

Hu, Y. \& M. Shum (2012) Nonparametric identification of dynamic models with unobserved state variables. Journal of Econometrics 171, 32-44.

Kasahara, H. \& K. Shimotsu (2009) Nonparametric identification of finite mixture models of dynamic discrete choices. Econometrica 77, 135-175.

Keane, M. \& K. Wolpin (2009) Empirical applications of discrete choice dynamic programming models. Review of Economic Dynamics 12, 1-22.

Kennet, D.M. (1994) A structural model of aircraft engine maintenance. Journal of Applied Econometrics 9, 351-368.

Klein, R. \& R. Spady (1993) An efficient semiparametric estimator for binary response models. Econometrica 61, 387-421.

Lewbel, A. (2000) Semiparametric qualitative response model estimation with unknown heteroscedasticity or instrumental variables. Journal of Econometrics 97, 145-177.

Lewbel, A. \& O. Linton (2007) Nonparametric matching and efficient estimators of homothetically separable functions. Econometrica 75, 1209-1227.

Lewbel, A. (2014) An overview of the special regressor method. In A. Ullah, J. Racine, \& L. Su (eds.), Oxford Handbook of Applied Nonparametric and Semiparametric Econometrics and Statistics, Oxford University Press, pp. 38-62.

Lewbel, A. \& X. Tang (2015) Identification and estimation of games with incomplete information using excluded regressors. Journal of Econometrics 189, 229-244.

Lippman, S. (1975) On dynamic programming with unbounded rewards. Management Science 21, $1225-1233$.

Magnac, T. \& D. Thesmar (2002) Identifying dynamic discrete decision processes. Econometrica 70, 801-816.

Manski, C.F. (1975) Maximum score estimation of the stochastic utility model of choice. Journal of Econometrics 3, 205-228.

Manski, C.F. (1985) Semiparametric analysis of discrete response: Asymptotic properties of the maximum score estimator. Journal of Econometrics 27, 313-333.

Manski, C.F. (1988) Identification of binary response models. Journal of the American Statistical Association 83, 729-738.

Manski, C.F. (1993) Dynamic choice in social settings: Learning from the experiences of others. Journal of Econometrics 58, 121-136.

Matzkin, R. (1991) Semiparametric estimation of monotone and concave utility functions for polychotomous choice models. Econometrica 59, 1315-1327.

Matzkin, R. (1992) Nonparametric and distribution-free estimation of the binary threshold crossing and the binary choice models. Econometrica 60, 239-270.

Matzkin, R. (1993) Nonparametric identification and estimation of polychotomous choice models. Journal of Econometrics 58, 137-168.

McKelvey, R.D. \& Palfrey, T.R. (1995) Quantal response equilibria for normal form games. Games and Economic Behavior 10, 6-38.

Miller, R. (1997) Estimating models of dynamic optimization with microeconomic data. In M. Pesaran \& P. Schmidt (eds.), Handbook of Applied Econometrics, vol. 2, Oxford: Blackwell Publishers, pp. 246-299.

Norets, A. (2010) Continuity and differentiability of expected value functions in dynamic discrete choice models. Quantitative Economics 1, 305-322.

Norets, A. \& X. Tang (2014) Semiparametric inference in dynamic binary choice models. Review of Economic Studies 81, 1229-1262.

Pakes, A., M. Ostrovsky, \& S. Berry (2007) Simple estimators for the parameters of discrete dynamic games (with entry/exit example). RAND Journal of Economics 38, 373-399. 
Pesendorfer, M. \& P. Schmidt-Dengler (2008) Asymptotic least squares estimator for dynamic games. Review of Economic Studies 75, 901-928.

Rust, J. (1987) Optimal replacement of GMC bus engines: An empirical model of Harold Zurcher. Econometrica 55, 999-1033.

Rust, J. (1988) Maximum likelihood estimation of discrete control processes. SIAM Journal on Control and Optimization 26, 1006-1024.

Rust, J. (1994) Structural estimation of Markov decision processes. In R. Engle \& D. McFadden (eds.), Handbook of Econometrics, vol. 4, pp. 3081-3143.

Sanches, F.A.M., D. Silva-Junior, \& S. Srisuma (2013) An Alternative Asymptotic Least Squares Estimator for Dynamic Games. Working paper, London School of Economics.

Sanches, F.A.M., D. Silva-Junior, \& S. Srisuma (2014) Identifying Dynamic Games with Switching Costs. Working paper, University of Surrey.

Srisuma, S. \& O. Linton (2012) Semiparametric estimation of Markov decision processes with continuous state space. Journal of Econometrics 166, 320-341.

Srisuma, S. (2015) Identification in discrete Markov decision models. Econometric Theory 31, 521-538.

Stokey, N. \& R. Lucas (1989) Recursive Methods in Economics Dynamics. Harvard University Press.

Taber, C. (2000) Semiparametric identification and heterogeneity in discrete choice dynamic programming models. Journal of Econometrics 96, 201-229.

Thompson, T.S. (1989) Identification of semiparametric discrete choice models. Discussion paper, University of Minnesota.

\section{APPENDIX A. Proofs of the theoretical results}

\section{A.1. Proof of Lemma 1}

Proof of Lemma 1. For part (i), let $M_{\varepsilon} \equiv E\left(\max _{j \in C}\left|\varepsilon_{j}^{\prime}\right|\right)$. By [M3], $M_{\varepsilon}<\infty$. Given [M2], we have

$$
\begin{aligned}
\left|E\left(\max _{j \in C}\left\{h_{j}\left(x^{\prime}\right)+\varepsilon_{j}^{\prime}\right\} \mid x, d=k\right)\right| & \leq E\left[\max _{j \in C}\left|h_{j}\left(x^{\prime}\right)+\varepsilon_{j}^{\prime}\right| \mid x, d=k\right] \\
& \leq E\left[\max _{j \in C}\left|h_{j}\left(x^{\prime}\right)\right| \mid x, d=k\right]+M_{\varepsilon} \\
& \leq\|h\|_{\alpha}(\alpha(x)+b)^{q}+M_{\varepsilon} .
\end{aligned}
$$

Since $\left(u_{k}^{*}\right)_{k \in C} \in B(\alpha, q)$, it follows that $\left|u_{k}^{*}(x)\right| \leq\left\|u^{*}\right\|_{\alpha} \alpha(x)^{q}$. Therefore, for each $k \in C$ and for each $x$,

$$
\left|\Gamma_{k}(h)(x)\right| \leq\left\|u^{*}\right\|_{\alpha} \alpha(x)^{q}+\beta\left[\|h\|_{\alpha}(\alpha(x)+b)^{q}+M_{\varepsilon}\right] .
$$

Since $\alpha(x) \geq 1, b>0$ and $h \in B(\alpha, q)$, it then follows that $\Gamma(h) \in B(\alpha, q)$.

For part (ii), by Denardo (1967), it suffices to establish that there is a positive integer $J$ such that the $J$-stage composition of $\Gamma$, denoted as $\Gamma^{J}$, defined on $B(\alpha, q)$ is a contraction mapping. We prove this by following closely the method of Lippman (1975). In particular, we also establish that for any positive integer $n$ and for $f, g \in B(\alpha, q)$,

$\left|\left(\Gamma_{k}^{n} f-\Gamma_{k}^{n} g\right)(x)\right| \leq \beta^{n}\|f-g\|_{\alpha}(\alpha(x)+n b)^{q}$ for $k \in C$.

To verify (A.1), note that, for each $k \in C$,

$$
\begin{aligned}
\left|\left(\Gamma_{k} f-\Gamma_{k} g\right)(x)\right| & \leq \beta E\left[\left|\max _{j \in C}\left\{f_{j}\left(x^{\prime}\right)+\varepsilon_{j}^{\prime}\right\}-\max _{j \in C}\left\{g_{j}\left(x^{\prime}\right)+\varepsilon_{j}^{\prime}\right\}\right| \mid x, d=k\right] \\
& \leq \beta E\left[\max _{j \in C}\left|f_{j}\left(x^{\prime}\right)-g_{j}\left(x^{\prime}\right)\right| \mid x, d=k\right] \\
& \leq \beta\|f-g\|_{\alpha}(\alpha(x)+b)^{q},
\end{aligned}
$$


where inequality (A.2) follows by using and interchanging $f_{j}$ and $g_{j}$ in the following inequality

$$
\max _{j \in C}\left\{f_{j}\left(x^{\prime}\right)+\varepsilon_{j}^{\prime}\right\} \leq \max _{j \in C}\left|f_{j}\left(x^{\prime}\right)-g_{j}\left(x^{\prime}\right)\right|+\max _{j \in C}\left\{g_{j}\left(x^{\prime}\right)+\varepsilon_{j}^{\prime}\right\} .
$$

We have shown that (A.1) holds for the case of $n=1$. The remaining inductive proof from step $n$ to step $n+1$ can now be completed by following the same arguments as those used in Lippman (1975, Lemma 1, pp. 1228-1229). Therefore, (A.1) holds for any positive integer $n$ by induction. Because the bound in (A.1) holds for all $k \in C$, given that $\alpha(x) \geq 1$ and $b>0$, it follows that $\left\|\Gamma_{k}^{J} f-\Gamma_{k}^{J} g\right\|_{\alpha} \leq \beta^{J}\|f-g\|_{\alpha}(1+J b)^{q}$ and thus we can choose $J$ sufficiently large such that $\beta^{J}(1+J b)^{q}<1$.

\section{A.2. Proof of Theorem 1}

We first give the following lemma, which will be invoked in the proofs of Theorem 1 and other theoretical results of the paper. Let $l(x)$ be a given scalar function. For $k \in C$, let $\Pi_{k}$ be the Bellman operator that maps a scalar function $f$ to another scalar function $\Pi_{k}(f)$ where

$\Pi_{k}(f)(x) \equiv l(x)+\beta E\left[f\left(x^{\prime}\right) \mid x, \delta=k\right]$.

Let $B_{\text {scalar }}(\alpha, q)$ be the space of scalar functions $f$ satisfying $\sup _{x}\left[|f(x)| \alpha(x)^{-q}\right]<\infty$, where $\alpha(x)$ and $q$ are the function and integer stated in assumption [M4], respectively.

LEMMA A.1. Assume that $l(x) \in B_{\text {scalar }}(\alpha, q)$. Then for $k \in C$, the following statements hold: (i) if $f \in B_{\text {scalar }}(\alpha, q)$, then $\Pi_{k}(f) \in B_{\text {scalar }}(\alpha, q)$. (ii) There is a unique scalar function $f^{*} \in B_{\text {scalar }}(\alpha, q)$ satisfying $f^{*}=\Pi_{k}\left(f^{*}\right)$.

Proof. Lemma A.1 follows immediately by simplifying the proof of Lemma 1 given in Appendix A.1.

We will also require the next lemma for the proof of Theorem 1 .

LEMMA A.2. Let $h=\left(h_{1}, \ldots, h_{K}\right)$ and $\widetilde{h}=\left(\widetilde{h}_{1}, \ldots, \widetilde{h}_{K}\right)$ be two $K$-dimensional real vectors such that $h_{K}=\widetilde{h}_{K}$ and $h_{j} \neq \widetilde{h}_{j}$ for at least one $j \in C \backslash\{K\}$. Then there is at least one $j \in C$ such that $G_{j}\left(\left(h_{j}-h_{k}\right)_{k \in C \backslash\{j\}}\right) \neq G_{j}\left(\left(\widetilde{h}_{j}-\widetilde{h}_{k}\right)_{k \in C \backslash\{j\}}\right)$.

Proof. Decompose the set $C=\{1, \ldots, K\}$ as $A \cup B \cup M$ where

$A \equiv\left\{j: h_{j}>\widetilde{h}_{j}\right\}, B \equiv\left\{j: h_{j}<\widetilde{h}_{j}\right\}, M \equiv\left\{j: h_{j}=\widetilde{h}_{j}\right\}$.

By assumption, $A \cup B \neq \varnothing$. We first consider the case where $B=\varnothing$. Since $G_{j}$ is strictly increasing in all its arguments and given $h_{K}=\widetilde{h}_{K}$, if $B=\varnothing$, Lemma A.2 follows by noting that

$G_{K}\left(\left(h_{K}-h_{k}\right)_{k \in C \backslash\{K\}}\right)<G_{K}\left(\left(\widetilde{h}_{K}-\widetilde{h}_{k}\right)_{k \in C \backslash\{K\}}\right)$.

Note that the proof for the case of $A=\varnothing$ can be completed using similar arguments. We now discuss the case where both $A$ and $B$ are non-empty. Since the cardinality of the set 
$A$ is finite, we may use the proof of Lemma 8 of Matzkin (1991, pp. 1324-1325) to deduce that there is a choice index $a \in A$ such that

$h_{a}-h_{k} \geq \widetilde{h}_{a}-\widetilde{h}_{k}$ for $k \in A \backslash\{a\}$.

Since $B \neq \varnothing$, we have that for any choice index $a^{\prime} \in A$,

$h_{a^{\prime}}-h_{k}>\widetilde{h}_{a^{\prime}}-\widetilde{h}_{k}$ for $k \in B$.

Hence Lemma A.2 follows by putting together (A.5) and (A.6) to deduce that

$G_{a}\left(\left(h_{a}-h_{k}\right)_{k \in C \backslash\{a\}}\right)>G_{a}\left(\left(\widetilde{h}_{a}-\tilde{h}_{k}\right)_{k \in C \backslash\{a\}}\right)$.

We now prove Theorem 1.

Proof of Theorem 1. Given $G$, we can deduce $G_{k}$ for all $k \in C \backslash\{K\}$ since $\left(\varepsilon_{j}-\varepsilon_{k}\right)_{j \in C \backslash\{k\}}$ can be expressed as a known linear transformation of $\left(\varepsilon_{j}-\varepsilon_{K}\right)_{j \in C \backslash\{K\}}$. For $j \in C$, let $\Delta v_{j}^{*} \equiv v_{j}^{*}-v_{K}^{*}$. Given $G_{k}$ for all $k \in C$, by (2.6) and Lemma A.2, the vector of value differences $\Delta v^{*} \equiv\left(\Delta v_{1}^{*}(x), \ldots, \Delta v_{K}^{*}(x)\right)$ can be identified as the unique vector $h=\left(h_{1}, \ldots, h_{K}\right)$ that simultaneously solves the equations $h_{K}=0$ and $P(\delta=j \mid x)=G_{j}\left(\left(h_{j}-h_{k}\right)_{k \in C \backslash\{j\}}\right)$ for all $j \in C$. Under assumption [M2] and given $G$ and identification of $\Delta v^{*}$, we can deduce the functions $\tau_{k}(x), k \in C$, where

$\tau_{k}(x) \equiv E\left[\max _{j \in C}\left\{\Delta v_{j}^{*}\left(x^{\prime}\right)+\Delta \varepsilon_{j}^{\prime}\right\} \mid x, \delta=k\right]$,

where $\Delta \varepsilon_{j} \equiv \varepsilon_{j}-\varepsilon_{K}$. Using equation (3.1), we have

$v_{K}^{*}(x)=l_{K}(x)+\beta E\left(v_{K}^{*}\left(x^{\prime}\right) \mid x, \delta=K\right)$,

where $l_{K}(x) \equiv u_{K}^{*}(x)+\beta \tau_{K}(x)$. By [M4], we have that $\sup _{x}\left[\left|u_{K}^{*}(x)\right| \alpha(x)^{-q}\right]<\infty$. By [M2], [M3] and [M4], using the proof of part (i) of Lemma 1, we see that both $\sup _{x}\left[\left|v_{K}^{*}(x)\right| \alpha(x)^{-q}\right]<\infty$ and

$\sup _{x}\left[\left|E\left(\max _{j \in C}\left\{v_{j}^{*}\left(x^{\prime}\right)+\varepsilon_{j}^{\prime}\right\} \mid x, \delta=k\right)\right| \alpha(x)^{-q}\right]<\infty$.

Thus by [M2], [M3] and the triangle inequality, we have that $\sup _{x}\left[\left|\tau_{k}(x)\right| \alpha(x)^{-q}\right]<\infty$ for $k \in C$. Given $\beta, u_{K}^{*}(x)$ and identification of $\tau_{K}(x)$, the function $l_{K}$ is identified and satisfies $\sup _{x}\left[\left|l_{K}(x)\right| \alpha(x)^{-q}\right]<\infty$. Hence by Lemma A.1, $v_{K}^{*}(x)$ can be identified as the unique fixed point of the Bellman equation (A.8). Since $v_{K}^{*}$ and $\Delta v^{*}$ are identified, the remaining value functions $v_{j}^{*}$ for $j \in C \backslash\{K\}$ can also be identified. Thus using (3.1), given $\beta$ and identification of $\tau_{k}, v_{k}^{*}$ and $v_{K}^{*}$, it follows that $u_{k}^{*}$ for $k \in C \backslash\{K\}$ can be identified as $u_{k}^{*}(x)=v_{k}^{*}(x)-\beta \tau_{k}(x)-\beta E\left(v_{K}^{*}\left(x^{\prime}\right) \mid x, \delta=k\right)$.

\section{A.3. Proof of Theorem 2}

Proof of Theorem 2. Under [M2] and assumption (ii), the function $m(x)$ does not depend on $z$. This together with assumption (i) implies that $\lambda(x)=\bar{\lambda}(w)$, which is a function of $w$ only. Hence the conditional choice probability equation (4.1) can be written as

$P(\delta=2 \mid w, z)=G\left(u_{2}^{*}(w, z)+\bar{\lambda}(w)\right)$. 
Let $G$ and $\bar{\lambda}$ denote the true data generating functions. Consider another function $\tilde{\lambda}(w)$ and distribution $\widetilde{G}$ that are compatible with the modeling assumptions. Let $M(\widetilde{\lambda})$ denote the event $\left\{w \in \Gamma_{W}: \widetilde{\lambda}(w) \neq \bar{\lambda}(w)\right\}$. We first establish the fact that, if $P(M(\widetilde{\lambda}))>0$, then $P(S(\widetilde{G}, \widetilde{\lambda}))>0$ as well where $S(\widetilde{G}, \widetilde{\lambda})$ denotes the event

$S(\widetilde{G}, \widetilde{\lambda}) \equiv\left\{(w, z) \in \Gamma_{X}: G\left(u_{2}^{*}(w, z)+\bar{\lambda}(w)\right) \neq \widetilde{G}\left(u_{2}^{*}(w, z)+\widetilde{\lambda}(w)\right)\right\}$.

This fact implies that, if the configurations $(G, \bar{\lambda})$ and $(\widetilde{G}, \tilde{\lambda})$ are observationally equivalent, then $\bar{\lambda}=\widetilde{\lambda}$ with probability 1 . Therefore the function $\bar{\lambda}$ is identified.

Suppose that $P(M(\widetilde{\lambda}))>0$. By assumption (iii), $G(0)=\widetilde{G}(0)=\tau$. Thus, to show $P(S(\widetilde{G}, \widetilde{\lambda}))>0$, it suffices to establish that the probability of the event $A(\widetilde{G}, \widetilde{\lambda}) \cup B(\widetilde{G}, \widetilde{\lambda})$ is positive where

$$
\begin{aligned}
& A(\widetilde{G}, \tilde{\lambda}) \equiv\left\{(w, z) \in \Gamma_{X}: u_{2}^{*}(w, z)+\bar{\lambda}(w) \geq 0>u_{2}^{*}(w, z)+\tilde{\lambda}(w)\right\} \\
& B(\widetilde{G}, \tilde{\lambda}) \equiv\left\{(w, z) \in \Gamma_{X}: u_{2}^{*}(w, z)+\bar{\lambda}(w)<0 \leq u_{2}^{*}(w, z)+\tilde{\lambda}(w)\right\} .
\end{aligned}
$$

Note that assumption (iv) implies that, for each $w \in M(\widetilde{\lambda})$,

$P\left(\bar{\lambda}(w) \geq-u_{2}^{*}(w, z)>\tilde{\lambda}(w) \mid w\right)+P\left(\bar{\lambda}(w)<-u_{2}^{*}(w, z) \leq \tilde{\lambda}(w) \mid w\right)>0$.

Since $P(M(\widetilde{\lambda}))>0$, it follows from inequality (A.10) that the probability of the set $A\left(\widetilde{u}_{2}, \widetilde{G}\right) \cup B\left(\widetilde{u}_{2}, \widetilde{G}\right)$ is also positive. Hence by the arguments above, the function $\bar{\lambda}$ is identified. Since $u_{2}^{*}(w, z)$ is known and $\bar{\lambda}$ is identified, observational equivalence between $G$ and $\widetilde{G}$ implies that $G(t)=\widetilde{G}(t)$ for each $t$ in the support of $u_{2}^{*}(w, z)+\bar{\lambda}(w)$. By assumption (iv), that support is the entire real line and thus $G(t)$ is identified at each $t \in R$.

\section{A.4. Proof of Theorem 3}

Proof of Theorem 3. Under [M2] and assumptions (i) and (ii) of Theorem 2, $\lambda(x)=\bar{\lambda}(w)$ is a function of $w$ only. Given $u_{2}^{*}(x)=\theta^{\prime} h(w, z)$, we have that

$P(\delta=2 \mid w, z)=G\left(\theta^{\prime} h(w, z)+\bar{\lambda}(w)\right)$.

Since $G$ is differentiable and $\partial h_{k}\left(w, z_{k}\right) / \partial z_{k}$ is almost surely non-zero, we have that

$$
\frac{\partial P(\delta=2 \mid w, z) / \partial z_{k}}{\partial h_{k}\left(w, z_{k}\right) / \partial z_{k}}=\theta_{k} g\left(\theta^{\prime} h(w, z)+\bar{\lambda}(w)\right)
$$

with probability 1 , where $g$ denotes the probability density function of $\varepsilon_{1}-\varepsilon_{2}$.

Using the average derivative arguments, we have

$\theta_{k}=E\left[\left(\frac{\partial P(\delta=2 \mid w, z) / \partial z_{k}}{\partial h_{k}\left(w, z_{k}\right) / \partial z_{k}}\right)\left(\frac{\partial h_{1}\left(w, z_{1}\right) / \partial z_{1}}{\partial P(\delta=2 \mid w, z) / \partial z_{1}}\right)\right] \operatorname{sign}\left(\theta_{1}\right)\left|\theta_{1}\right|$.

By (A.11), the sign of $\theta_{1}$ is the same as that of $E\left[\left(\partial P(\delta=2 \mid w, z) / \partial z_{1}\right) /\right.$ $\left.\left(\partial h_{1}\left(w, z_{1}\right) / \partial z_{1}\right)\right]$. Given that $\left|\theta_{1}\right|=1$, it therefore follows that $\theta_{k}$ is identified for $k \in\{1, \ldots, J\}$. 


\section{A.5. Proof of Theorem 4}

Proof of Theorem 4. The proof of this theorem is essentially the same as that of Theorem 2. To avoid repetition, we retain all the notation defined in the proof of Theorem 2. Note that by assumption (iv*), we have that for each $w \in M(\widetilde{\lambda})$, either

$$
P\left(0 \leq u_{2}^{*}(w, z)+\bar{\lambda}(w)<\bar{\lambda}(w)-\tilde{\lambda}(w) \mid w\right)>0
$$

or

$$
P\left(0>u_{2}^{*}(w, z)+\bar{\lambda}(w) \geq \bar{\lambda}(w)-\tilde{\lambda}(w) \mid w\right)>0 .
$$

Therefore, inequality (A.10) also holds and, by the same arguments as those used in the proof of Theorem 2, the function $\lambda(x)=\bar{\lambda}(w)$ is identified. By equation (4.2) and given $u_{2}^{*}$, the function $v_{2}^{*}-v_{1}^{*}$ is also identified. Using equation (4.1), we have

$G(t)=P\left(\delta=2 \mid v_{2}^{*}(x)-v_{1}^{*}(x)=t\right)$.

Therefore, $G(t)$ is also identified at each $t \in \Omega_{12}$.

\section{A.6. Proof of Theorem 5}

Proof of Theorem 5. Under [M2] and assumption (ii), it follows that, for all $j \in C \backslash\{K\}$, $m_{j}(x)=\bar{m}_{j}(w)$, which is a function of $w$ only. This together with assumption (i) implies that $\lambda_{1}(x)=\bar{\lambda}_{1}(w)$, which is a function of $w$ only, and for $j \in\{2, \ldots, K-1\}, \lambda_{j}(x)=$ $\bar{\lambda}_{j}\left(w, z_{j-1}\right)$, which is a function of $\left(w, z_{j-1}\right)$ only. Let

$\bar{\lambda}\left(w, z_{(K-1)}\right) \equiv\left(\bar{\lambda}_{1}(w), \bar{\lambda}_{2}\left(w, z_{1}\right), \ldots, \bar{\lambda}_{K-1}\left(w, z_{K-2}\right)\right)$

denote the vector of true data generating functions. Consider another vector of functions

$\tilde{\lambda}\left(w, z_{(K-1)}\right) \equiv\left(\tilde{\lambda}_{1}(w), \tilde{\lambda}_{2}\left(w, z_{1}\right), \ldots, \tilde{\lambda}_{K-1}\left(w, z_{K-2}\right)\right)$

such that $P(M(\tilde{\lambda}))>0$ where

$M(\widetilde{\lambda}) \equiv\left\{\left(w, z_{(K-1)}\right) \in \Gamma_{W} \times \Gamma_{Z_{(K-1)}}: \bar{\lambda}\left(w, z_{(K-1)}\right) \neq \tilde{\lambda}\left(w, z_{(K-1)}\right)\right\}$.

Using similar arguments to those given in the proof of Theorem 2, we will show that $\tilde{\lambda}$ and $\lambda$ do not generate the same conditional choice probabilities. This fact implies that, if $\widetilde{\lambda}$ and $\lambda$ are observationally equivalent, then $\bar{\lambda}=\widetilde{\lambda}$ with probability 1 . Therefore the vector of functions $\bar{\lambda}$ is identified.

For $j \in C \backslash\{K\}$, define the events

$A_{j}(\widetilde{\lambda}) \equiv\left\{(w, z) \in \Gamma_{X}: \bar{v}_{K j}^{*}(w, z)>0>\widetilde{v}_{K j}^{*}(w, z)\right.$ or $\left.\bar{v}_{K j}^{*}(w, z)<0<\widetilde{v}_{K j}^{*}(w, z)\right\}$,

where $\bar{v}_{K 1}^{*}(w, z) \equiv \bar{u}_{K}^{*}\left(w, z_{K-1}\right)+\bar{\lambda}_{1}(w)$ and $\widetilde{v}_{K 1}^{*}(w, z) \equiv \bar{u}_{K}^{*}\left(w, z_{K-1}\right)+\widetilde{\lambda}_{1}(w)$ and, for $j \in\{2, \ldots, K-1\}, \bar{v}_{K j}^{*}(w, z) \equiv \bar{u}_{K}^{*}\left(w, z_{K-1}\right)+\bar{\lambda}_{j}\left(w, z_{j-1}\right)$ and $\widetilde{v}_{K j}^{*}(w, z) \equiv$ $\bar{u}_{K}^{*}\left(w, z_{K-1}\right)+\widetilde{\lambda}_{j}\left(w, z_{j-1}\right)$. By Condition 1 and equation (5.1), whenever the event $\cup_{j \in C \backslash\{K\}} A_{j}(\widetilde{\lambda})$ occurs, the conditional choice probabilities induced by $\bar{\lambda}$ are ranked differently from those induced by $\widetilde{\lambda}$. Hence, for identification of $\bar{\lambda}$, it suffices to show that the condition $P(M(\widetilde{\lambda}))>0$ implies $P\left(\cup_{j \in C \backslash\{K\}} A_{j}(\widetilde{\lambda})\right)>0$. 
Note that for each $\left(w, z_{(K-1)}\right) \in M(\widetilde{\lambda})$, there is some index $j^{*} \in C \backslash\{K\}$ such that the value of $\bar{\lambda}_{j^{*}}$ differs from that of $\widetilde{\lambda}_{j^{*}}$ at the point $\left(w, z_{(K-1)}\right)$. By assumption (iii), if $j^{*} \in$ $\{2, \ldots, K-1\}$, then

$P\left(\bar{\lambda}_{j^{*}}\left(w, z_{j^{*}-1}\right)>-\bar{u}_{K}^{*}\left(w, z_{K-1}\right)>\widetilde{\lambda}_{j^{*}}\left(w, z_{j^{*}-1}\right) \mid w, z_{(K-1)}\right)+$

$P\left(\bar{\lambda}_{j^{*}}\left(w, z_{j^{*}-1}\right)<-\bar{u}_{K}^{*}\left(w, z_{K-1}\right)<\widetilde{\lambda}_{j^{*}}\left(w, z_{j^{*}-1}\right) \mid w, z_{(K-1)}\right)>0$.

If $j^{*}=1$, we have

$P\left(\bar{\lambda}_{1}(w)>-\bar{u}_{K}^{*}\left(w, z_{K-1}\right)>\tilde{\lambda}_{1}(w) \mid w, z_{(K-1)}\right)+$

$P\left(\bar{\lambda}_{1}(w)<-\bar{u}_{K}^{*}\left(w, z_{K-1}\right)<\tilde{\lambda}_{1}(w) \mid w, z_{(K-1)}\right)>0$.

Since

$P\left(\cup_{j \in C \backslash\{K\}} A_{j}(\widetilde{\lambda}) \mid w, z_{(K-1)}\right) \geq \max _{j \in C \backslash\{K\}} P\left(A_{j}(\widetilde{\lambda}) \mid w, z_{(K-1)}\right)$,

inequalities (A.12) and (A.13) imply that $P\left(\cup_{j \in C \backslash\{K\}} A_{j}(\widetilde{\lambda}) \mid w, z_{(K-1)}\right)>0$ for each $\left(w, z_{(K-1)}\right) \in M(\widetilde{\lambda})$. Since $P(M(\widetilde{\lambda}))>0, P\left(\cup_{j \in C \backslash\{K\}} A_{j}(\widetilde{\lambda})\right)$ is thus also positive. It then follows that the functions $\bar{\lambda}_{j}, j \in C \backslash\{K\}$, are identified.

By (5.1), we have that, for $j \in C \backslash\{1\}$,

$v_{1}^{*}(x)-v_{j}^{*}(x)=\bar{\lambda}_{j}\left(w, z_{j-1}\right)-\bar{\lambda}_{1}(w)=\bar{m}_{j}(w)-\bar{\lambda}_{1}(w)-\bar{u}_{j}^{*}\left(w, z_{j-1}\right)$.

Therefore, by assumption (iv), the support of $\left(v_{1}^{*}(x)-v_{j}^{*}(x)\right)_{j \in\{2, \ldots, K\}}$ conditional on $w=c$ is the entire $R^{K-1}$. Given identification of all $\bar{\lambda}_{j}$, we can hence identify $G_{1}(t)$ at each $t \in R^{K-1}$ since

$G_{1}(t)=P\left(\delta=1 \mid w=c,\left(\bar{\lambda}_{j}\left(w, z_{j-1}\right)-\bar{\lambda}_{1}(w)\right)_{j \in C \backslash\{1\}}=t\right)$.

Thus the distribution $G=G_{K}$ is also identified by noting that the vector $\left(\varepsilon_{j}-\varepsilon_{K}\right)_{j \in C \backslash\{K\}}$ can be expressed as a known linear transformation of $\left(\varepsilon_{j}-\varepsilon_{1}\right)_{j \in C \backslash\{1\}}$.

\section{A.7. Proof of Theorem 6}

Proof of Theorem 6. Under [M2] and assumptions (i) and (ii) of Theorem 5, for all $j \in C \backslash\{K\}, m_{j}(x)=\bar{m}_{j}(w)$, which is a function of $w$ only. Moreover, $\lambda_{1}(x)=\bar{\lambda}_{1}(w)$, which is a function of $w$ only, and for all $j \in C \backslash\{1\}, \lambda_{j}(x)=\bar{\lambda}_{j}\left(w, z_{j-1}\right)$, which is a function of $\left(w, z_{j-1}\right)$ only. Hence, by (5.1) and given $u_{K}^{*}(x)=\theta^{\prime} h\left(w, z_{K-1}\right)$, we have

$v_{1}^{*}(x)-v_{K}^{*}(x)=-\theta^{\prime} h\left(w, z_{K-1}\right)-\bar{\lambda}_{1}(w)$

and, for $j \in\{2, \ldots, K-1\}$,

$v_{1}^{*}(x)-v_{j}^{*}(x)=\bar{\lambda}_{j}\left(w, z_{j-1}\right)-\bar{\lambda}_{1}(w)$.

Therefore, by (2.6),

$P(\delta=1 \mid w, z)$
$\quad=G_{1}\left(\bar{\lambda}_{2}\left(w, z_{1}\right)-\bar{\lambda}_{1}(w), \ldots, \bar{\lambda}_{K-1}\left(w, z_{K-2}\right)-\bar{\lambda}_{1}(w),-\theta^{\prime} h\left(w, z_{K-1}\right)-\bar{\lambda}_{1}(w)\right)$. 
Note that the variables $\left(z_{j, K-1}\right)_{j=1, \ldots, J}$ only appear in the last argument of $G_{1}$ in the equation above. The rest of the proof hence proceeds along the same lines as those for the proof of Theorem 3. By the average derivative arguments, it is then straightforward to see that

$\theta_{k}=E\left[\left(\frac{\partial P(\delta=1 \mid w, z) / \partial z_{k, K-1}}{\partial h_{k}\left(w, z_{k, K-1}\right) / \partial z_{k, K-1}}\right)\left(\frac{\partial h_{1}\left(w, z_{1, K-1}\right) / \partial z_{1, K-1}}{\partial P(\delta=1 \mid w, z) / \partial z_{1, K-1}}\right)\right] \operatorname{sign}\left(\theta_{1}\right)\left|\theta_{1}\right|$.

The sign of $\theta_{1}$ is the same as that of $-E\left[\left(\partial P(\delta=1 \mid w, z) / \partial z_{1, K-1}\right) /\left(\partial h_{1}\left(w, z_{1, K-1}\right) /\right.\right.$ $\left.\left.\partial z_{1}, K-1\right)\right]$. Given $\left|\theta_{1}\right|=1$, it therefore follows that $\theta_{k}$ is identified for $k \in\{1, \ldots, J\}$.

\section{A.8. Proof of Theorem 7}

Proof of Theorem 7. The proof of this theorem is essentially the same as that of Theorem 5. To avoid repetition, we retain all the notation defined in the proof of Theorem 5. We first establish identification of $\underset{\tilde{\lambda}}{\bar{\lambda}}\left(w, z_{(K-1)}\right)$ by showing that the condition $P(M(\widetilde{\lambda}))>0$ implies that $P\left(\cup_{j \in C \backslash\{K\}} A_{j}(\widetilde{\lambda})\right)>0$.

Note that for each $(w, z(K-1)) \in M(\widetilde{\lambda})$, there is some index $j^{*} \in C \backslash\{K\}$ such that the value of $\bar{\lambda}_{j^{*}}$ differs from that of $\widetilde{\lambda}_{j^{*}}$ at the point $\left(w, z_{(K-1)}\right)$. By assumption (iii*), if $j^{*} \in\{2, \ldots, K-1\}$, then either

$P\left(\bar{\lambda}_{j^{*}}\left(w, z_{j^{*}-1}\right)-\widetilde{\lambda}_{j^{*}}\left(w, z_{j^{*}-1}\right)>\bar{u}_{K}^{*}\left(w, z_{K-1}\right)+\bar{\lambda}_{j^{*}}\left(w, z_{j^{*}-1}\right)>0 \mid w, z_{(K-1)}\right)>0$

or

$P\left(\bar{\lambda}_{j^{*}}\left(w, z_{j^{*}-1}\right)-\widetilde{\lambda}_{j^{*}}\left(w, z_{j^{*}-1}\right)<\bar{u}_{K}^{*}\left(w, z_{K-1}\right)+\bar{\lambda}_{j^{*}}\left(w, z_{j^{*}-1}\right)<0 \mid w, z_{(K-1)}\right)>0$.

If $j^{*}=1$, we have that either

$P\left(\bar{\lambda}_{1}(w)-\tilde{\lambda}_{1}(w)>\bar{u}_{K}^{*}\left(w, z_{K-1}\right)+\bar{\lambda}_{1}(w)>0 \mid w, z_{(K-1)}\right)>0$

or

$P\left(\bar{\lambda}_{1}(w)-\widetilde{\lambda}_{1}(w)<\bar{u}_{K}^{*}\left(w, z_{K-1}\right)+\bar{\lambda}_{1}(w)<0 \mid w, z_{(K-1)}\right)>0$.

Therefore inequalities (A.12) and (A.13) also hold and, by the same arguments as those used in the proof of Theorem 5, the vector of functions $\bar{\lambda}$ is identified. Given (5.1), the vector of value differences $\left(v_{K}^{*}(x)-v_{j}^{*}(x)\right)_{j \in C \backslash\{K\}}$ is thus also identified. By (2.6), it follows that the function $G(t)=G_{K}(t)$ is identified at every $t \in \Omega_{K}$.

\section{APPENDIX B. Derivation of the bounds of the structural value functions when the value difference exhibits insufficient variation}

We consider the binary choice setup of Section 4.1. Recall that the set $\Omega_{12}$ is the support of $v_{2}^{*}(x)-v_{1}^{*}(x)$. The proof of Theorem 4 implies that $v_{2}^{*}(x)-v_{1}^{*}(x)=u_{2}^{*}(x)+\lambda(x)$ is 
identified and the distribution $G(t)$ is identified at each $t \in \Omega_{12}$. Assume that $\Omega_{12}$ is a bounded and connected interval. Let $[L, U]$ denote this interval. For $k \in\{1,2\}$, define

$\tau_{k}(x) \equiv E\left[\max \left\{v_{1}^{*}\left(x^{\prime}\right)-v_{2}^{*}\left(x^{\prime}\right)+\varepsilon_{1}^{\prime}-\varepsilon_{2}^{\prime}, 0\right\} \mid x, \delta=k\right]=c_{k}(x)+e_{k}(x)$

where

$c_{k}(x) \equiv E\left[1\left\{\delta^{\prime}=1\right\}\left(v_{1}^{*}\left(x^{\prime}\right)-v_{2}^{*}\left(x^{\prime}\right)\right) \mid x, \delta=k\right]$,

$e_{k}(x) \equiv E\left[1\left\{\delta^{\prime}=1\right\}\left(\varepsilon_{1}^{\prime}-\varepsilon_{2}^{\prime}\right) \mid x, \delta=k\right]$.

Note that the functions $c_{k}(x)$ are identified because the value difference is identified. The functions $e_{k}$ are not point identified but their lower bounds can be derived. By assumption [M2], we have

$e_{k}(x)=E\left[\varphi\left(x^{\prime}\right) \mid x, \delta=k\right]+\kappa$

where

$\varphi\left(x^{\prime}\right) \equiv \int_{v_{2}^{*}\left(x^{\prime}\right)-v_{1}^{*}\left(x^{\prime}\right)}^{U} t d G(t), \kappa \equiv \int_{U}^{\infty} t d G(t)$.

Since $G$ is only identified on $\Omega_{12}$, the term $\kappa$ is not identified but can be bounded below by $U(1-G(U))$. Define $l(x) \equiv u_{2}^{*}(x)+\beta \tau_{2}(x)$. It then follows that $l(x) \geq \underline{l}(x)$ for each $x$ where

$\underline{l}(x) \equiv u_{2}^{*}(x)+\beta\left[c_{k}(x)+E\left[\varphi\left(x^{\prime}\right) \mid x, \delta=k\right]+U(1-G(U))\right]$.

By assumption [M4], we have that $\sup _{x}\left[\left|u_{2}^{*}(x)\right| \alpha(x)^{-q}\right]<\infty$. Note that $\left|\varphi\left(x^{\prime}\right)\right| \leq$ $E\left(\left|\varepsilon_{1}^{\prime}\right|+\left|\varepsilon_{2}^{\prime}\right|\right)$. Hence, by [M3] with [M4] and using the proof of part (i) of Lemma 1, we see that $\sup _{x}\left[\underline{l}(x) \mid \alpha(x)^{-q}\right]<\infty$. Hence, by Lemma A.1 of Appendix A.2, there is a unique function $\underline{v}$ satisfying the Bellman equation

$\underline{v}(x)=\underline{l}(x)+\beta E\left(\underline{v}\left(x^{\prime}\right) \mid x, \delta=2\right)$.

Given $\beta, \underline{l}(x)$ is identified and thus $\underline{v}$ can be identified by solving the functional equation (B.1). By equation (3.1), we have

$v_{2}^{*}(x)=l(x)+\beta E\left(v_{2}^{*}\left(x^{\prime}\right) \mid x, \delta=2\right)$.

Since $l \geq \underline{l}$ and the function $v_{2}^{*}-v_{1}^{*}$ is identified by $u_{2}^{*}+\lambda$, separate identifiable lower bounds can be obtained for the value functions $v_{1}^{*}$ and $v_{2}^{*}$ using the following inequalities:

$v_{1}^{*} \geq \underline{v}-\left(u_{2}^{*}+\lambda\right)$

$v_{2}^{*} \geq \underline{v}$. 